\title{
A Beginner's Guide to the Modern Theory of Polarization
}

\author{
Nicola A. Spaldin \\ Materials Theory, ETH Zurich, Wolfgang-Pauli-Strasse 27, 8093 Zürich, Switzerland
}

\begin{abstract}
The so-called Modern Theory of Polarization, which rigorously defines the spontaneous polarization of a periodic solid and provides a route for its computation in electronic structure codes through the Berry phase, is introduced in a simple qualitative discussion.
\end{abstract}

Keywords: polarization, Berry phase, electronic structure calculation

\section{Introduction}

The concept of electric dipole moment is central in the theory of electrostatics, particularly in describing the response of systems to applied electric fields. For finite systems such as molecules it poses no conceptual or practical problems. In the ionic limit the dipole moment, $d$, of a collection of charges, $q_{i}$, at positions $\mathbf{r}_{i}$ is defined as

$$
d=\Sigma_{i} q_{i} \mathbf{r}_{i}
$$

for the case of a continuous charge density, en $(\mathbf{r})$ (where $e$ is the electronic charge and $n(\mathbf{r})$ is the number density) this expression is straightforwardly extended to

$$
d=\int e n(\mathbf{r}) \mathbf{r} d \mathbf{r}
$$

Provided that the molecule or cluster carries no net charge these expressions are well defined, can be straightforwardly evaluated and yield results - for example for the direction of the dipole moment - that are consistent with our intuitive understanding.

Things apparently start to turn to custard, however, when we try to extend this simple reasoning to bulk solids. The usual way to define intrinsic 
quantities in macroscopic systems is to introduce the property per unit volume or mass. For example the magnetization is the magnetic moment per unit volume, and the bulk analogue to the electric dipole moment, the electric polarization, should be represented by the electric dipole moment per unit volume. The relevant quantity is then evaluated within a small repeat unit - the unit cell - of the solid, and normalized with the volume of the chosen unit cell. The problem with this simple method in the case of electric polarization can be understood in the simple one-dimensional cartoon of Figure 1: Without performing any calculations, we can see that the two equally valid unit cells shown with dashed lines have completely opposite orientations of the polarization!

This difficulty led to tremendous confusion in the field, with discussions as fundamental as whether the polarization (and related quantities such as the piezoelectric response) could be considered as intrinsic properties in bulk solids, or are in fact determined by details of the surface termination. Thankfully the confusion was resolved around 20 years ago with the introduction of the so-called Modern theory of polarization. This very elegant theory showed that changes in polarization are in fact rigorously defined, can be calculated quantum mechanically using electronic structure methods, and correspond to experimentally measurable observables.

The purpose of this article is to introduce in the simplest possible terms the apparent difficulties associated with defining polarization in bulk solids, and the solutions provided by the modern theory. It is motivated by my having explained these concepts repeatedly to many and diverse students ranging from experimentalists with a casual interest in understanding obscure theory papers to beginning hard-core theoretical solid-state physicists and quantum chemists. This article in no way intends to substitute for the elegant early papers on the topic, nor the subsequent detailed and rigorous review papers which are referenced throughout. Indeed I hope that this informal introduction provides sufficient background for the reader to tackle these excellent articles without intimidation.

\section{Bulk periodicity, the polarization lattice and the polarization quantum}

We begin by reconciling the different values for polarization obtained for the different choices of unit cells in Fig. 1 by introducing a formal concept that at first sight is even more confusing - that is the multi-valuedness of the 
bulk polarization. We will show, however, that a multi-valued polarization is a natural consequence of the periodicity in a bulk solid, and hopefully that it is actually not so frightening. We will see, in fact that changes in polarization - which are the quantities that are anyway measured in experiments - are single valued and well defined, and we can once again sleep without anxiety.

We take the simplest possible example of a one-dimensional chain of singly charged alternating anions and cations - the closest real-life analogue would be rock-salt structure sodium chloride in just one direction. Look at Figure 1 which shows such a chain with the atoms spaced a distance $a / 2$ apart so that the lattice constant is $a$. The first thing to notice is that all of the ions are centers of inversion symmetry: If I sit on any ion and look to the left, then to the right I see no difference. So by definition this lattice is non-polar.

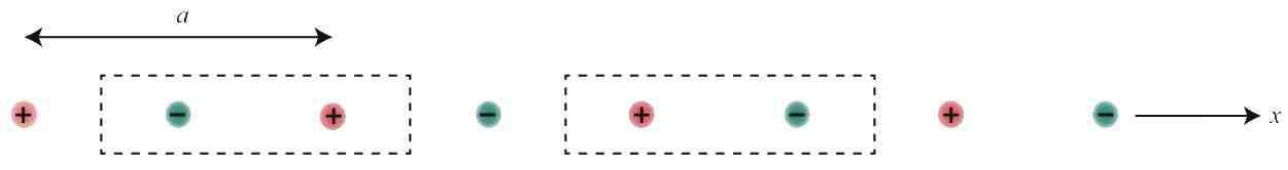

Figure 1: One-dimensional chain of alternating anions and cations, spaced a distance $a / 2$ apart, where $a$ is the lattice constant. The dashed lines indicate two representative unit cells which are used in the text for calculation of the polarization.

Now let's work out the polarization by calculating the dipole moment per unit length (the definition in three dimensions is dipole moment per unit volume) using in turn the two unit cells shown as the dashed rectangles to compute the local dipole moment. First, the cell on the left. Taking the left edge of the shell as the origin $(x=0)$, we have an ion with charge -1 at position $a / 4$, and an ion with charge +1 at position $3 a / 4$. So the polarization, or dipole moment per unit length is

$$
\begin{aligned}
p & =\frac{1}{a} \sum_{i} q_{i} x_{i} \\
& =\frac{1}{a}\left(-1 \times \frac{a}{4}+1 \times \frac{3 a}{4}\right) \\
& =\frac{1}{a} \frac{2 a}{4} \\
& =\frac{1}{2}
\end{aligned}
$$


in units of $|e|$ per unit length. Immediately we have an apparent problem: Using this method, our non-polar chain has a non-zero polarization.

I am afraid that things will get worse before they get better. Next, let's do the same exercise using the right-most unit cell. Again taking the left edge of the unit cell as the origin, this time there is a positively charged ion at position $a / 4$, and a negatively charged ion at $3 a / 4$. So

$$
\begin{aligned}
p & =\frac{1}{a}\left(+1 \times \frac{a}{4}-1 \times \frac{3 a}{4}\right) \\
& =\frac{1}{a} \times-\frac{2 a}{4} \\
& =-\frac{1}{2} .
\end{aligned}
$$

Again a non-zero value, and this time different from the value we obtained using the other, equally valid unit cell, by an amount $a$.

So what is going on here, and how can we connect it to physical reality? Well, if we were to repeat this exercise with many choices of unit cell (convince yourself by choosing a couple of arbitrary unit cells and giving it a try!), we would obtain many values of polarization, with each value differing from the original value by an integer. We call this collection of polarization values the polarization lattice. In this case it is $\ldots,-5 / 2,-3 / 2,-1 / 2,1 / 2,3 / 2,5 / 2 \ldots$. Notice that the lattice of polarization values is symmetric about the origin. In fact this is the signature of a non-polar structure: The polarization lattice may or may not contain zero as one of its elements, but it must be centrosymmetric around zero.

Now what is the significance of the spacing (in this case 1) between the allowed values? Well, imagine removing an electron from one of the anions in the lattice (leaving a neutral atom) and moving it by one unit cell to put it on the next anion to the right. Because of the periodic boundary conditions of the infinite lattice, the next anion simultaneously has it's electron removed and moved one unit cell to the right, and so it is able to accept the incoming electron and appear unchanged at the end of the process. There has been no change in the physics of the system resulting from the relocation of the electrons by one unit cell to the right. But what has happened to the polarization? Well, in each unit cell a charge of -1 has moved a distance $a$, changing the dipole moment by $-a$ and the polarization by -1 . We can clearly perform this thought experiment any number of times, and in either direction, changing the polarization by any integer without changing the physical 
system! We call the value of polarization resulting from moving one electron by one unit cell the polarization quantum, $P_{q}$. In one dimension it is equal to the lattice constant divided by the length of the unit cell, which is just an integer (in units of the electronic charge per unit length). Going back to the polarization lattice of our non-polar chain, we see that it's polarization values correspond to half-polarization quanta. In fact all non-polar systems have polarization lattices of either $0 \pm n P_{q}$ or $\frac{P_{q}}{2} \pm n P_{q}$.

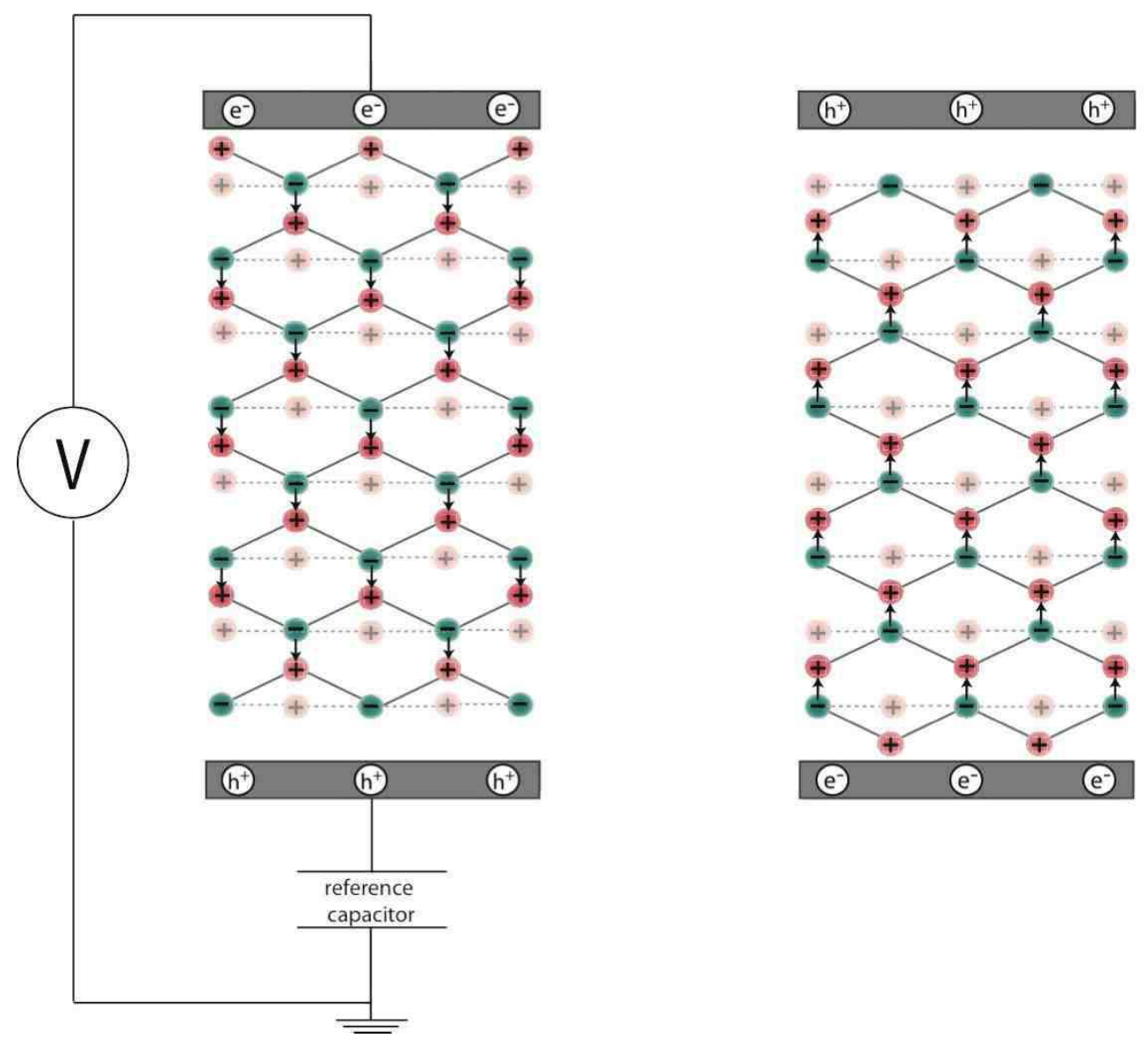

Figure 2: Schematic of the Sawyer-Tower method of measuring ferroelectric polarization. The material on the left is polarized in the up direction and its surface charge is screened by electrons in the upper electrode (grey) and holes in the lower electrode. When the polarization is switched (right), electrons and holes flow through the external circuit to screen the new opposite surface charges, and are counted by comparing the voltage across the material with that across a reference capacitor. 
If this all seems too esoteric, please bear with me for one more paragraph by which time I hope things should start making sense. First, let's think about how we measure electrical polarization, and what a reported measured polarization really means. Look at Figure 2 - this is a cartoon of a standard way of measuring the electrical polarization using a so-called Sawyer-Tower circuit. On the left the material has become polarized in the up direction as a result of the cation sub-lattice displacing upwards relative to the anion sub-lattice. This could happen, for example during a ferroelectric phase transition with an external electric field applied in the up direction (the light colored cations with the dashed-line bonds indicate their positions in the high-symmetry, paraelectric structure). Electrons accumulate at the upper electrode, and holes (or a depletion of electrons) at the lower electrode in order to screen the surface charge resulting from the ionic displacements. In fact, on each electrode, the accumulated charge per unit area is exactly equal to the polarization of the sample. So if we could measure the amount of charge accumulation we would have a direct measure of the polarization. But how can we do this? Well, next, imagine reversing the orientation of the polarization - for example by applying an external electric field in the down direction - to reach the configuration on the right. Now electrons accumulate at the lower electrode and holes at the upper electrode to achieve the screening. They achieve this by flowing through the external circuit connecting the two electrodes, where they can be counted by comparing the voltage across the series reference capacitor then using $Q=C V$ ! The amount of charge per unit area of electrode that flows during the transition is equal to the change in polarization between the up- and down-polarized states; the "absolute" value of polarization which is reported is half of this number.

Now, bearing in mind that what is measured in an experiment is a change in polarization, let's go back to our cartoon one-dimensional model and make some sense out of this multi-valuedness business. In the upper part of Figure 3 we reproduce the non-polar one-dimensional chain of Fig. 1, and below it we show a similar chain in which the cations have been displaced by a distance $d$ relative to the anions in the manner of a ferroelectric distortion to create a polar system. Let's repeat our earlier exercise of calculating the polarization using the two unit cells shown as the dashed rectangles. 


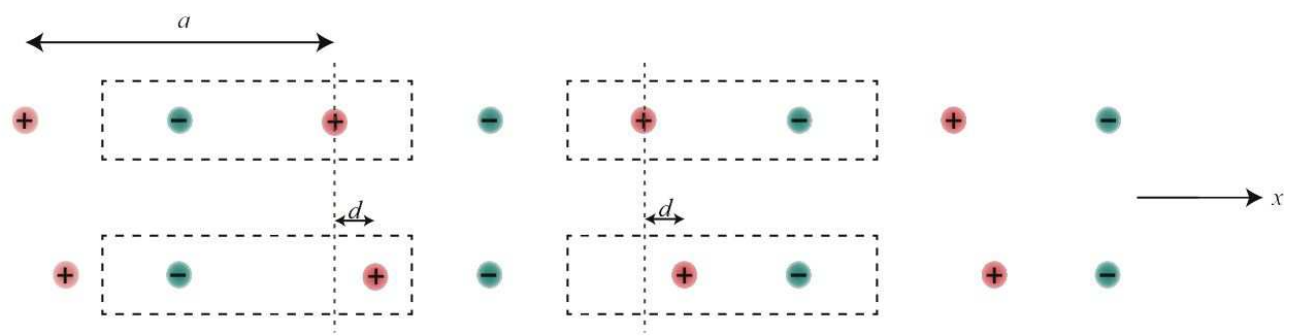

Figure 3: The upper panel reproduces the one-dimensional chain of alternating anions and cations of Fig. 1. In the lower panel, the cations are displaced to the right by a distance $d$ relative to the anions, with the vertical dotted lines indicating their original positions.

In the left hand case,

$$
\begin{aligned}
p=\frac{d}{a} & =\frac{1}{a} \sum_{i} q_{i} x_{i} \\
& =\frac{1}{a}\left(-1 \times \frac{a}{4}+1 \times\left(\frac{3 a}{4}+d\right)\right) \\
& =\frac{1}{2}+\frac{d}{a}
\end{aligned}
$$

and in the right hand case,

$$
\begin{aligned}
p & =\frac{1}{a}\left(+1 \times\left(\frac{a}{4}+d\right)-1 \times \frac{3 a}{4}\right) \\
& =-\frac{1}{2}+\frac{d}{a} .
\end{aligned}
$$

Again the two answers are different, but this time that doesn't worry us, because we recognize that they differ by exactly one polarization quantum. Next comes the key point: Let's calculate the change in polarization between the polar and non-polar chains using each unit cell as our basis. First for the cell on the left,

$$
\delta p=\left(\frac{1}{2}+\frac{d}{a}\right)-\frac{1}{2}=\frac{d}{a}
$$

and for the cell on the right

$$
\delta p=-\left(-\frac{1}{2}+\frac{d}{a}\right)-\left(-\frac{1}{2}\right)=\frac{d}{a} .
$$


In both cases the change in polarization between polar and non-polar chains is the same. In fact this would have been the case whatever unit cell we had chosen to make the calculation. So, while the absolute value of polarization in a bulk, periodic system, is multivalued, the change in polarization - which remember is the quantity that can be measured in an experiment - is single valued and well defined. Phew.

Just to really drive the point home, in Fig. 4 we plot the polarization of the ideal one-dimensional ionic chain as a function of the displacement of the cations (as a fraction of the lattice constant) from their non-polar positions. As we calculated earlier, for zero displacement the polarization lattice is centrosymmetric and consists of all half-integer values (black circles). As the displacement increases, the polarization increases linearly and by the same amount along each branch of the polarization lattice (labeled by $n=-1,0,1$ etc.) The branches are always separated from each other by the same amount, the polarization quantum, which is equal to 1 in this case. The dashed lines on the $n=1$ branch show that for a displacement of $0.25 a$, the polarization increases from 0.5 to 0.75 , and so the change in polarization is 0.25 . If the displacement is increased artificially to 0.5 - that is half of the unit cell - the ions end up on top of each other; in our thought experiment this causes the polarization to jump between branches of the polarization lattice, although in practice we would have achived nuclear fusion which would likely dominate the physics.

\subsection{Extension to three dimensions}

The one-dimensional example that we chose here for simplicity is not entirely without physical relevance; for example ferroelectric or polar polymers closely resemble one-dimensional chains. Such an application is discussed in Ref. [1], along with an excellent analysis of the development of an infinite chain from a finite one. In most cases, however, we are interested in threedimensional systems, and fortunately the extension to three dimensions is

straightforward: A polarization lattice can now be defined along all three lattice vector directions, with the polarization quantum equal to

$$
P_{i}=\frac{1}{\Omega} e R_{i}
$$

Here $e$ is the electronic charge, $R_{i}$ is the $i$ th lattice vector, and $\Omega$ is the unit cell volume. Note that in non-magnetic systems, the polarization quantum is 


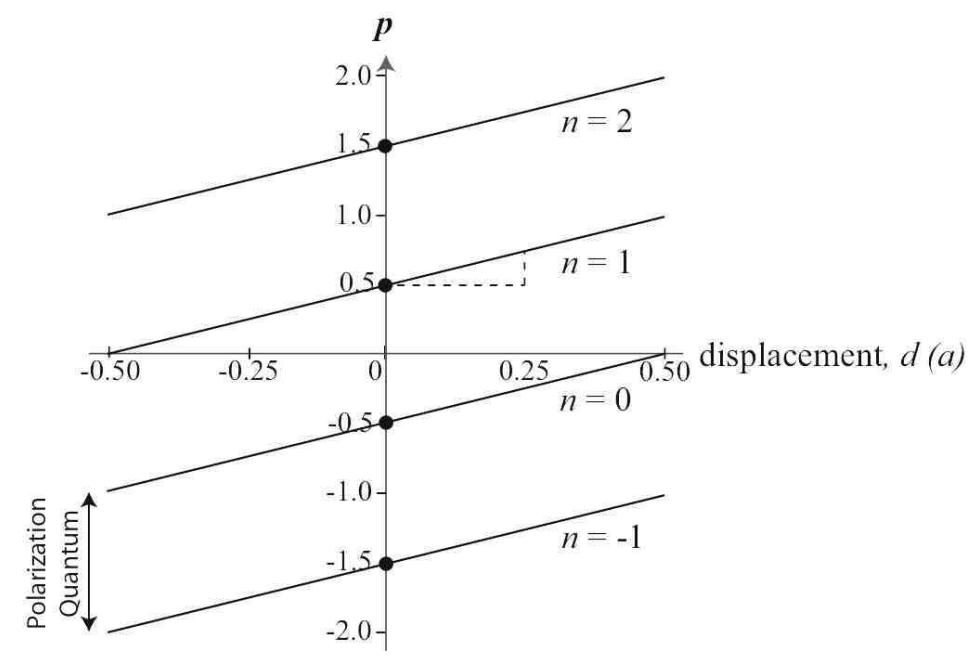

Figure 4: Polarization as a function of the displacement, $d$, of the cations in the 1D chain of Figure 3. The polarization lattice is zero-centered, and the branches are separated by the polarization quantum. Notice that the branches of the lattice run exactly parallel to each other, so that differences in polarization along each branch for the same displacement are identical.

usually multiplied by an additional factor of two because the up- and downspin electrons are equivalent, and shifting an up-spin electron by a lattice vector also shifts the corresponding down-spin electron. Polarization values along cartesian coordinates, for example, can then be readily obtained using the appropriate linear transformation.

\section{Wannier representation and Berry Phase}

In the previous section we discussed the multivaluedness of the polarization in a bulk periodic solid and reconciled it with what can be measured experimentally for the simple example of an array of ions. Of course in a real solid, there is (thankfully) more chemistry to take care of. In this section we will first explain how this chemistry can be incorporated rather simply by extending the ionic model through the method of Wannier functions. (A similar approach is followed in Ref. [2], where an algorithm is developed that is particularly suited to localized-basis quantum chemistry codes.) Once we are comfortable with this conceptually we will move on to the real meat of the modern theory of polarization - the Berry phase method. 
Remember that the Wannier function, $w_{n}(\mathbf{r})$, in unit cell $\mathbf{R}$ associated with band $n$ is defined as

$$
\begin{aligned}
w_{n}(\mathbf{r}-\mathbf{R}) & =\frac{\Omega}{(2 \pi)^{3}} \int_{B Z} d^{3} \mathbf{k} e^{-i \mathbf{k} \cdot \mathbf{R}} \Psi_{n \mathbf{k}}(\mathbf{r}) \\
& =\frac{\Omega}{(2 \pi)^{3}} \int_{B Z} d^{3} \mathbf{k} e^{i \mathbf{k} \cdot(\mathbf{r}-\mathbf{R})} u_{n \mathbf{k}}(\mathbf{r})
\end{aligned}
$$

where $\Psi_{n \mathbf{k}}(\mathbf{r})=e^{i \mathbf{k} \cdot \mathbf{r}} u_{n \mathbf{k}}(\mathbf{r})$ are the Bloch functions, written as usual in terms of the cell-periodic part, $u_{n \mathbf{k}}(\mathbf{r})$. Here $\Omega$ is the unit cell volume, and the integral is over the Brillouin zone.

Unlike the Bloch functions which are delocalized in space, the Wannier functions are localized. As a result they are often used in visualization of chemical bonding, as well as for basis sets in electronic structure calculations, where their minimal overlap can lead to favorable scaling with system size. They are relevant here, because their localized nature provides a convenient atomic-like description of the charge density in a solid: While we know in reality that the charge density in a solid is a continuous function, the localized picture will allow us to continue to calculate dipole moments by summing over charges multiplied by positions.

Let's go back to our 1D chain, and relax the constraint that it is composed of point charge ions to give it some chemistry. If it's helpful you could think of it as say a chain of $\mathrm{Na}^{+}$cations alternating with $\mathrm{Cl}^{-}$anions. In the following figures we associate pink with $\mathrm{Na}$ ions or electrons, and green with $\mathrm{Cl}$ ions or electrons. In Figure 5 (left) we show the molecular orbitals that would form between two such ions in an Na-Cl "molecule" - the lower energy, bonding orbital is occupied by two electrons and more localized on the $p$ orbital of the anion, and the higher energy, antibonding orbital is empty and consists primarily of cation $s$ character. The corresponding band structure cartoon is shown to the right; you can derive the dispersion using simple linearcombination-of-atomic-orbitals (LCAO) methods; see for example the book by Cox [3]. In Figure 6] we show a cartoon of our 1D chain again, but this time we have separated out the charge on the ions (all of which are +1 , and which we continue to treat as point charges) from the charge on the electrons which are spread through the system, but piled up more on the anions than the cations. The blobs around the anions illustrate what we might expect the Wannier functions of the occupied band to look like, with each Wannier function containing two electrons. The character of the Wannier function is mostly $\mathrm{Cl} p$-like, with a little bit of $\mathrm{Na} s$ character, indicated by the slight 
pink tinge on the edges. Note that if we consider both of the electrons in each Wannier function to be associated with the $\mathrm{Cl}$ ion, then the formal charge on the $\mathrm{Cl}$ is +1 (the ionic charge) $-2=-1$, and that on the $\mathrm{Na}$ ion is $+1+0$ $=+1$, and we recover our simple ionic model of Figs. 1 and 3 .
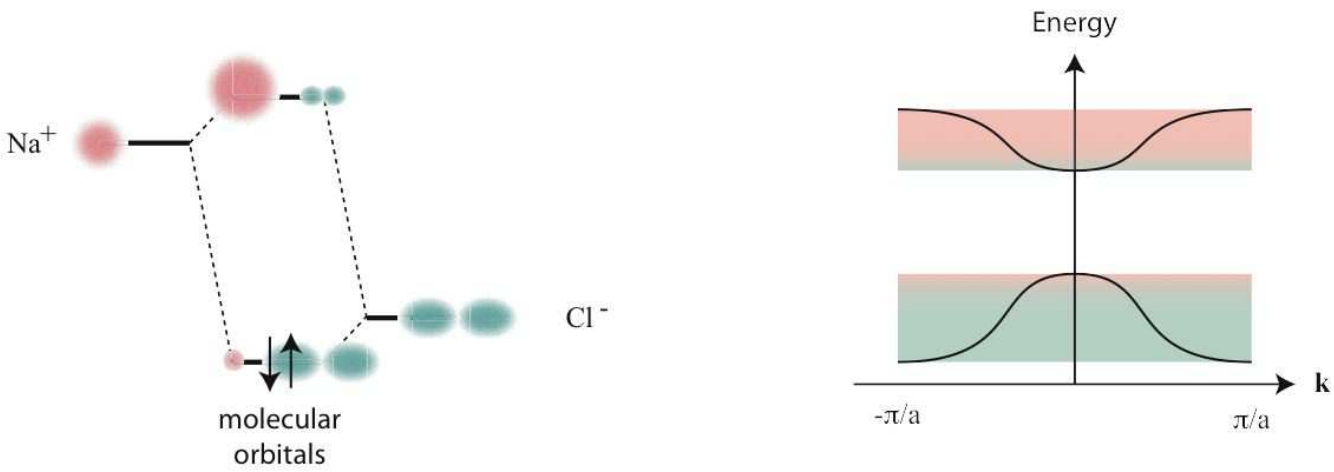

Figure 5: Left: The molecular orbitals formed in an Na-Cl "molecule". Right: Band structure of a one-dimensional $\mathrm{Na}-\mathrm{Cl}$ chain. The valence band is derived from Cl-like molecular orbitals each containing two electrons, and is fully occupied; the Na-like conduction band is empty. In both cases pink represents Na-derived states and green Cl-derived states.

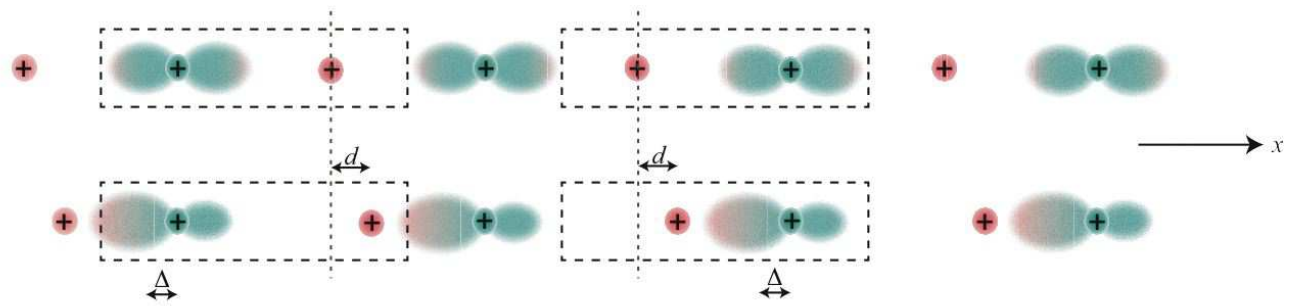

Figure 6: One-dimensional chain of alternating cations (pink postively charged ion cores) and anions (green positively charged ion cores with their associated negatively charged valence electron cloud). The dimensions and dashed unit cells are as in Figure 1.

How should we now calculate the polarization of the chain? We would like again to reduce our polarization integral again to a sum over localized charges multiplied by their positions. This is straightforward for the ions 
which we are still treating as point charges. For the electrons, it turns out that this procedure will work too. Since the Wannier functions are localized, we work out the average position of the electrons in the Wannier function, and treat them all as sitting at that point. This "position" of the Wannier function is called the Wannier center, $\overline{\mathbf{r}}_{n}$. The Wannier center associated with band $n$ is defined to be the expectation value of the position operator $\mathbf{r}$ for Wannier function $w_{n}(\mathbf{r})$ :

$$
\overline{\mathbf{r}}_{n}=\int w_{n}^{*}(\mathbf{r}) \mathbf{r} w_{n}(\mathbf{r}) d^{3} \mathbf{r}
$$

Later we will find it useful to rewrite this expression in terms of the periodic cell functions using the momentum representation of the position operator $\mathbf{r}=-i \frac{\partial}{\partial \mathbf{k}}:$

$$
\overline{\mathbf{r}}_{n}=i \frac{\Omega}{(2 \pi)^{3}} \int_{B Z} d^{3} \mathbf{k} e^{-i \mathbf{k} \cdot \mathbf{R}}\left\langle u_{n \mathbf{k}} \mid \frac{\partial u_{n \mathbf{k}}}{\partial \mathbf{k}}\right\rangle
$$

You can spend your next free Sunday morning showing that Eqns. 11 and 12 are equivalent, take my word for it, or follow the derivation by Blount in Ref. [4]

With this concept of the Wannier center, the expression for polarization that we used previously for the ionic chain extends simply to a sum over the contribution from the point charge ions, plus a sum over the electronic charges centered at the Wannier centers of each occupied Wannier function, $n$ :

$$
p=\frac{1}{a}\left(\sum_{i}\left(q_{i} x_{i}\right)^{i o n s}+\sum_{n}^{o c c}\left(q_{n} \overline{\mathbf{r}}_{n}\right)^{W F s}\right)
$$

Let's try it for the case of the left-hand unit cell in our 1D chain. In the non-polar case, we can see by symmetry that the Wannier center is at the same position as the green anion; remember now also that the charge on all of the ions is +1 , and that each Wannier function contains two electrons. So the dipole moment per unit length in the left unit cell is

$$
\begin{aligned}
p & =\frac{1}{a}\left(+1 \times \frac{a}{4}+1 \times \frac{3 a}{4}+-2 \times \frac{a}{4}\right) \\
& =\frac{1}{a} \frac{2 a}{4} \\
& =\frac{1}{2} .
\end{aligned}
$$


The same result as we obtained previously! This is as expected - the allowed values of the polarization lattice for a centrosymmetric structure are dictated by the symmetry of the crystal and the ionic charges, and are not modified by factors such as the details of the chemical bonding within the material.

Now let's think about the off-centered case, in the lower part of Fig. 6. As before, the cations have moved a distance $d$ to the right, but this time the Wannier centers have also moved - by a distance $\Delta$ say - to the left. This occurs as the chemical bond between the near neighbor anion-cation pairs becomes stronger, and develops more cation $s$ character, whereas that between the distant neighbor pairs weakens; you can think of it as a flow of electrons from the anion (which previously had all of the valence electrons) towards the cation in the process of covalent bond formation. Let's see what this additional covalency does to the polarization:

$$
\begin{aligned}
p & =\frac{1}{a}\left(+1 \frac{a}{4}+1\left(\frac{3 a}{4}+d\right)+-2\left(\frac{a}{4}-\Delta\right)\right) \\
& =\frac{1}{a}\left(\frac{2 a}{4}+d+2 \Delta\right) \\
& =\frac{1}{2}+\frac{d}{a}+2 \frac{\Delta}{a} .
\end{aligned}
$$

Compared to the purely ionic case, the polarization has increased by an amount $\frac{2 \Delta}{a}$. This is because, in addition to the positively charged cation moving to the right (along the positive $x$ axis) some negatively charged electron density has moved to the left (along the negative $x$ axis). This results in a larger effective displacement of positive charge along $+x$ and a larger polarization. We'll return to this picture later when we discuss the concept of the Born effective charge.

Let's summarize the discussion so far before we go on to formalize it mathematically. Using our Wannier function picture we can continue to write our polarization as the sum over the charges times their positions. We include both the contribution from the postively charged ion cores, and the contribution from the negatively charged valence electrons, and we take the "position" of each valence electron to be its Wannier center. Had we repeated our analysis for the right-hand unit cell, we would have seen that, as in the purely ionic model, the polarization is multi-valued, but the difference in polarization for example between a centrosymmetric and polar structure, is well-defined, and corresponds to the experimentally measurable spontaneous polarization. 
Now we will derive the formal mathematical expression for the spontaneous polarization $\delta p$ in the Wannier representation. Since we already have an expression for the Wannier centers this is going to be rather painless. Remember that the spontaneous polarization is the difference in polarization, on the same branch of the polarization lattice, between the final, polarized and initial, unpolarized states. Using $p=\frac{1}{\Omega} \sum_{i} q_{i} \mathbf{r}_{i}$ for the ionic part, and Eqn. 12 for the Wannier centers, we obtain:

$$
\begin{aligned}
\delta p & =p^{f}-p^{0} \\
& =\frac{1}{\Omega} \sum_{i}\left[q_{i}^{f} \mathbf{r}_{i}^{f}-q_{i}^{0} \mathbf{r}_{i}^{0}\right] \\
& -\frac{2 i e}{(2 \pi)^{3}} \sum_{n}^{o c c}\left[\int_{B Z} d^{3} \mathbf{k} e^{-i \mathbf{k} \cdot \mathbf{R}}\left\langle u_{n \mathbf{k}}^{f} \mid \frac{\partial u_{n \mathbf{k}}^{f}}{\partial \mathbf{k}}\right\rangle-\left\langle u_{n \mathbf{k}}^{0} \mid \frac{\partial u_{n \mathbf{k}}^{0}}{\partial \mathbf{k}}\right\rangle\right]
\end{aligned}
$$

where $f$ and 0 indicate the final (polar) and intial (high symmetry) positions/wavefunctions. Since the wavefunctions, at least at the Kohn-Sham level, are a direct output of standard electronic structure codes, Eqn. 16 can be used to evaluate the polarization with only a small extension to a standard density functional theory code. (A rigorous extension to correlated, many-body wavefunctions also exists, see for example Refs. [5] and [6].) Notice of course, that the issues discussed earlier about multivaluedness of the polarization and the polarization lattice persist here, and in taking the difference in Eqn. 16 one must be careful to remain on the same branch of the polarization lattice.

If you are familiar with the concept of the Berry phase [7] and its extension to periodic solids [8] you will recognize the integrals in Eqn. 16] to be the Berry phase developed by the wavefunction $u_{n \mathbf{k}}$ as it evolves along the path k. As a result, the formalism for calculating polarization using this method is often called the Berry phase theory of polarization. Refs. [9, 10, 11, 12] are the original papers providing the detailed derivations of the Berry phase formalism, and excellent reviews can be found in Refs. [13, 14, 15]. If you find the Berry phase concept too frightening, however, just stick with the Wannier function ideas, and regard Eqn. 16 as a tool that we'll see in Section 5 allows for convenient computation.

\subsection{Subtlety - gauge transformation!}

Those of you who have managed to stay awake and alert to this point might raise an objection: Since the Bloch functions are defined only to within 
a phase factor, i.e.

$$
\Psi_{n \mathbf{k}}(\mathbf{r}) \rightarrow e^{i \phi(\mathbf{k})} \Psi_{n \mathbf{k}}(\mathbf{r})
$$

without changing any physically meaningful quantities, the Wannier functions are not uniquely defined! As a result, the Wannier centers, which we have just seen are crucial in defining the polarization, are also not uniquely defined. We are saved, however, by the fact that the sum over the Wannier centers in any given unit cell is uniquely defined, and looking again at Eqn. 13 we find that this is in fact the quantity that matters in defining the polarization. In practice, special choices of Wannier functions are often made in calculations of the polarization. The so-called Maximally-localized Wannier functions in which the phases of the Bloch functions are chosen so as to minimize the sum of the mean squares of the positional spread [16] are particularly popular.

\section{The concept of Born Effective Charge}

At this stage I think it's appropriate to formally introduce the Born effective charge, which is a quantity that is very useful conceptually in thinking about ferroelectric polarization. In fact we have already seen the main idea, in Section 3, where we saw that the polarization resulting from the displacement of an ion could be different from that expected by multiplying its formal charge times its displacement, in the case when the Wannier center(s) move by a different amount than the ion cores. In fact in the example of Fig. 6, as the positive cations moved to the right, the Wannier centers shifted to the left, resulting in a larger overall polarization than we would have expected from the formal charges alone. We say that the effective charges on the ions

- the amount of charge that effectively contributes to the polarization during the displacement - is larger than the formal charge.

This is formalized in the concept of the Born effective charge, $Z^{*}$, which is defined as the change in polarization divided by the amount that an ion (or rather the periodic sub-lattice of equivalent ions) is displaced:

$$
Z_{i j}^{*}=\frac{\Omega}{e} \frac{\delta P_{i}}{\delta d_{j}} .
$$

The Born effective charge is a tensor: When an ionic sublattice is displaced in direction $i$, there is of course a change in polarization along the displacement direction, but in addition, the polarization in perpendicular directions, $j$, 
can change. Turning this expression around we can see immediately what we have been discussing qualitatively - that the change in polarization is determined by these effective charges times their displacements, not by the formal charges:

$$
\partial P_{i}=\frac{e}{\Omega} Z_{i j}^{*} \delta d_{j}
$$

The total polarization is then obtained by summing over the contributions from the displacements of all sublattices.

In materials that are ferroelectric, or that are close to a ferroelectric phase transition, the Born effective charges tend to be anomalously large, particularly on the atoms that displace the furthest from their high symmetry to their ferroelectric positions. For example in the prototypical ferroelectric $\mathrm{PbTiO}_{3}$, in which the formal charges are $\mathrm{Pb}+2, \mathrm{Ti}+4$ and $\mathrm{O} 2-$, the effective charges on the ions that are active during the ferroelectric phase transition are $\mathrm{Pb}+3.9, \mathrm{Ti}+7.1$ and $\mathrm{O}-5.8$ [17]. This is consistent, with the alternative, equivalent definition of the Born effective charge as the force induced on an ion by a uniform small electric field, $E$ :

$$
Z_{i j}^{*}=-e \frac{\delta F_{i}}{\delta E_{j}}
$$

In highly polarizable ferroelectrics, small electric fields generate large forces on the ions, mediated by the anomalously large Born effective charges.

Lastly, I want to emphasize that it is important to distinguish between the Born effective charge, which is a well-defined dynamical and measurable quantity, and the formal, static charge on an ion. The latter quantity, which reflects the number of electrons sitting at a particular ion site, depends on how you "count", since there is not a unique way of deciding how to apportion the electrons in a chemical bond to one ion or another. While the static charge indeed indicates a measure of the amount of covalency in a compound, it is not a good indicator of ferroelectricity, which is rather indicated by a change in covalency during ionic displacement.

\section{A few tips on getting a Berry Phase calculation to work}

Finally we describe a few of the tricks and foibles that we have learned through (sometimes) bitter experience are needed to make a Berry phase calculation of the polarization both run and give the correct answer. We try 
to keep our comments general - for the specifics of a particular code refer to the relevant manual.

The first step is of course to calculate the structure (if required) and selfconsistent charge density, as in any standard total energy calculation. Of course the charge density should be well-converged with respect to the energy cutoff and k-point sampling. In addition, if one is interested in systems such as improper ferroelectrics with small polarization values [18, 19], the ionic positions must be obtained with higher-than-usual accuracy. An extra subtlety is to check that the system is insulating at every point in $\mathbf{k}$-space, otherwise the Berry phase is ill-defined. The relaxed ionic positions and self-consistent charge density are then used as an input to the Berry phase calculation.

One then proceeds to calculate one of the Berry phase values on the right-hand side of Eqn. 16, that is

$$
\sum_{n}^{o c c} \int_{B Z} d^{3} \mathbf{k} e^{-i \mathbf{k} \cdot \mathbf{R}}\left\langle u_{n \mathbf{k}} \mid \frac{\partial u_{n \mathbf{k}}}{\partial \mathbf{k}}\right\rangle,
$$

where $u_{n \mathbf{k}}$ is the cell part of the Bloch function for the structure we are considering. First, the matrix elements are calculated by integrating along strings of $k$-points. Since $\frac{\partial}{\partial \mathbf{k}}$ is a vector derivative the matrix elements should be computed along any three non-collinear directions; usually the lattice vectors are chosen. Then multiple strings in a particular direction are sampled so that an integration over the Brillouin zone can be performed (see Figure 7). It's important to check convergence both with respect to the number of $k$-points along a string, and the number of strings used in the sampling, as the requirements can be quite different in each case. Finally the values for all bands $n$ are summed. One subtletly, which is sometimes not well taken care of in codes, concerns the procedure for averaging the Berry phase over the Brillouin zone. This is usually done by taking the sum of the Berry phase values at each $k$-point, weighted by the fractional contribution of the $k$-point. This procedure works well provided that the value from each $k$-point is on the same branch of the polarization lattice. Figure 8 illustrates a not-uncommon problem that can occur with some codes during the averaging procedure. Here by inspection the average Berry phase is clearly close to $\pi$, modulo the phase quantum of $2 \pi$. Taking a simple average of the values mapped into the range between $\pm \pi$, however, would result in an incorrect value close to zero. We recommend checking the values of Berry phase obtained for the individual strings if your code performs an automatic averaging procedure! 
For a spin-polarized system, the Berry phase calculation is performed for both up- and down-spin electrons separately; the phases are converted into polarization units by multiplying by $-\frac{i e}{2 \pi^{3}}$ and then added to the ionic contribution $\frac{1}{\Omega} \sum_{i} q_{i} \mathbf{r}_{i}$, where $q_{i}$ is the charge of the pseudopotential or ion, to obtain the total polarization of the system along the chosen lattice vector.

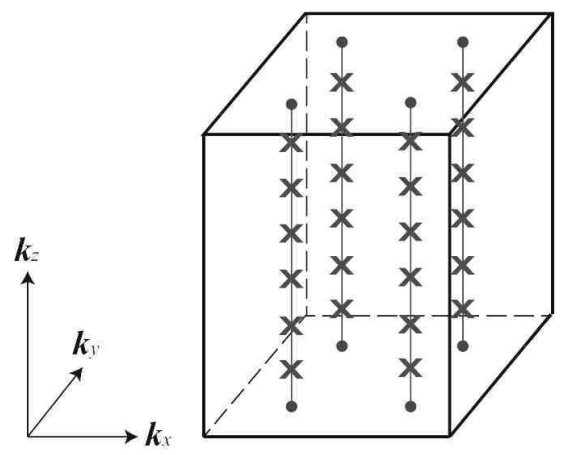

Figure 7: Choice of the k-point grid for a Berry phase calculation of the polarization. Here the polarization is to be calculated along the $z$-direction. The integration to obtain the Berry phase is carried out along 4 strings of $\mathbf{k}$ points centered around $\Gamma$ in the $\mathbf{k}_{x}-\mathbf{k}_{y}$ plane, with 6 sampling points along each string in the $\mathbf{k}_{z}$ direction. The final Berry phase is obtained by averaging the values obtained from each of the four strings.

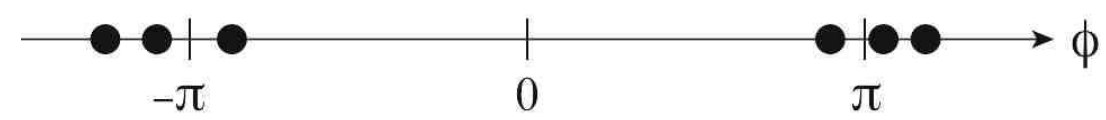

Figure 8: The black dots show the values of Berry phase obtained by integration along six k-point strings in the Brillouin zone. Clearly the average value is close to $\pi, \pm$ the Berry phase quantum of $2 \pi$. Mapping all of the values into the lowest phase branch then taking the simple average would lead to an incorrect result close to zero.

The above procedure is repeated for each lattice vector in turn. Be careful to check in the output of your code whether the results are reported with respect to the lattice vectors or in cartesian coordinates!

Remember that the number that you have now calculated is the absolute value of the polarization, and is only defined modulo a polarization quantum. To calculate the spontaneous polarization in a ferroelectric for example, the procedure should be repeated also for a high symmetry, non-polar reference state. The difference between the two values, taken along the same branch of the polarization lattice, is then the spontaneous polarization. Sometimes 
it is necessary to re-calculate the polarization for a number of structures along the deformation path between the high- and low-symmetry structures in order to know unambiguously which difference to take. For example, Fig. 9 shows the calculated polarization values for the case of perovskite structure $\mathrm{BiFeO}_{3}$, one of the most well-studied multiferroic materials[20]. Notice first that the polarization lattice for the non-polar structure, labeled with $0 \%$ distortion, does not contain zero, but is centered around $92.8 \mu \mathrm{C} \mathrm{cm}^{-2}$, which is half a polarization quantum. It is clear from following the evolution of the polarization with distortion that the correct value for the spontaneous polarization is $187.8-92.8=95.0 \mu \mathrm{C} \mathrm{cm}^{-2}$. From a calculation of only the end-points at the $R 3 c$ and $-R 3 c$ structures the appropriate difference to take would be unclear, and one might incorrectly assume a value of $\frac{1}{2}(2.3-$ $(-2.3))=2.3 \mu \mathrm{C} \mathrm{cm}^{-2}$.

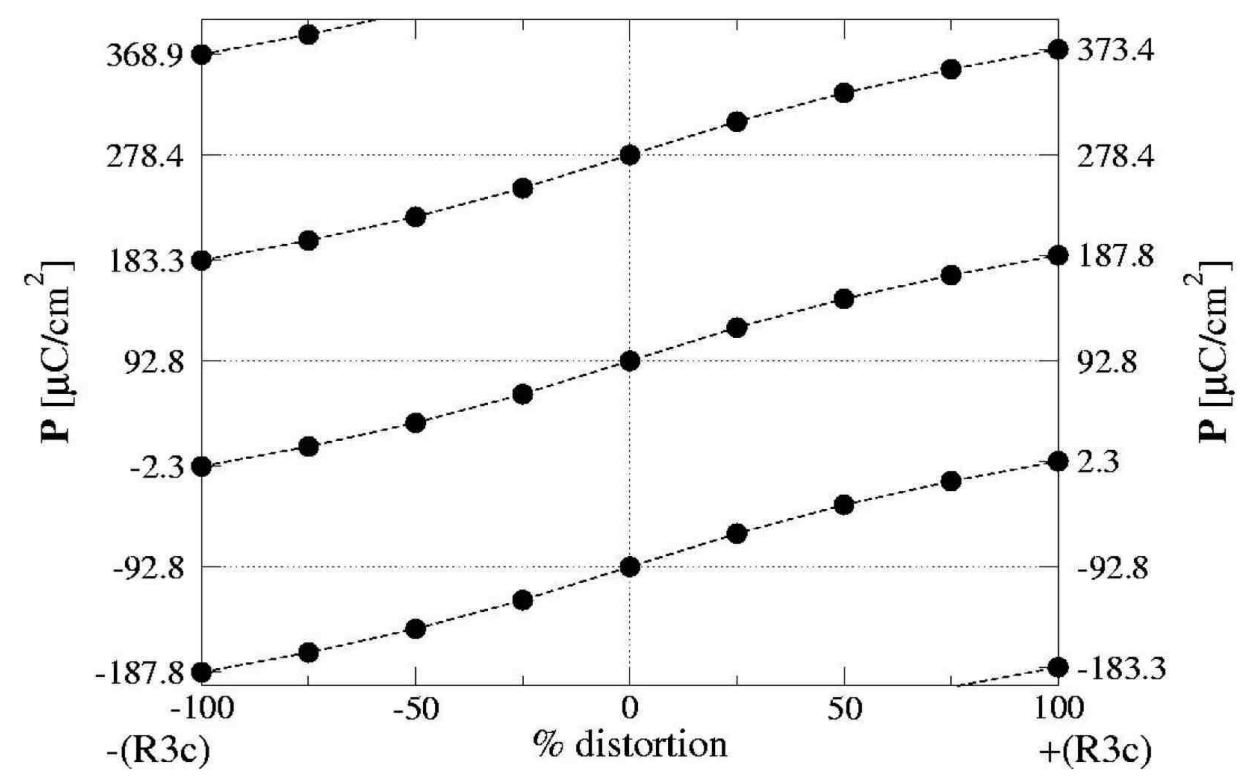

Figure 9: Calculated polarization as a function of percentage distortion from the high symmetry non-polar structure ( $0 \%$ distortion) to the ground state $R 3 c$ structure for perovskite $\mathrm{BiFeO}_{3}$. The black dots are calculated points and the dashed lines are a guide to the eye illustrating the evolution along branches of the polarization lattice. From Ref. 20].

Finally a hint for calculating Born Effective Charges. Since these are defined as derivatives, in principle the polarization should be calculated for the structure of interest, and then again for an infinitesimally small displacement 
of each ion in turn. In practice, however, if the diplacement is too small the result from this approach can be noisy. The best plan is to plot polarization as a function of ionic displacement, starting with very small displacement values, and to take the slope of the line in the region beyond the noise but before the non-linear regime.

\section{Last words}

I hope that this article has taken away some of the mystique associated with the modern theory of polarization, and motivated you to start making your own calculations of spontaneous polarization and related dielectric properties. For more practical introductory help, I recommend working through the tutorials that accompany many of the electronic structure computational packages. For example the Lesson on polarization and finite electric field provided by the ABINIT code, www . abinit.org, is particularly helpful. Or even better, attend a hands-on course hosted by one of the public codes where you will have direct access to leading experts in the field. Good luck!

\section{Acknowledgements}

My thanks to the pioneers of the Modern Theory of Polarization - Raffaele Resta and David Vanderbilt - who helped me to understand their elegant theory, as well as to the many students who have allowed me to impose my explanations upon them and in turn improve my description. The preparation of the manuscript was supported by ETH Zürich.

\section{References}

[1] K. N. Kundin, R. Car, R. Resta, J. Chem. Phys. 127 (2007) 194902.

[2] K. N. Kundin, R. Car, R. Resta, J. Chem. Phys. 126 (2007) 234101.

[3] P. A. Cox, The electronic structure and chemistry of solids, Oxford University Press, 1987.

[4] E. I. Blount, Solid State Physics 13 (1962) 305.

[5] R. Resta, Int. J. Quant. Chem. 75 (1999) 599.

[6] R. Resta, Phys. Rev. Lett. 80 (1998) 1800. 
[7] M. V. Berry, Proc. Roy. Soc. Lond. A 392 (1984) 45-57.

[8] J. Zak, Phys. Rev. Lett. 62 (1989) 2747.

[9] R. D. King-Smith, D. Vanderbilt, Phys. Rev. B 47 (1993) R1651-R1654.

[10] R. D. King-Smith, D. Vanderbilt, Phys. Rev. B 49 (1994) 5828-5844.

[11] R. Resta, Eur. Phys. Lett. 22 (1993) 133-138.

[12] R. Resta, Rev. Mod. Phys. 66 (1994) 899-915.

[13] R. Resta, Troisième Cycle de la Physique en Suisse Romande (Année Acade'emique 1995-96).

[14] R. M. Martin, Electronic structure: Basic Theory and Practical Methods, Cambridge University Press, 2004.

[15] R. Resta, D. Vanderbilt, Topics Appl. Physics 105 (2007) 31-68.

[16] N. Marzari, D. Vanderbilt, Phys. Rev. B 56 (1997) 12847-12865.

[17] P. Ghosez, J.-P. Michenaud, X. Gonze, Phys. Rev. B 58 (1998) 62246239.

[18] T. Kimura, T. Goto, H. Shintani, K. Ishizaka, T. Arima, Y. Tokura, Nature 426 (2003) 55-58.

[19] A. Malashevich, D. Vanderbilt, Phys. Rev. Lett. 101 (2008) 037210.

[20] J. B. Neaton, C. Ederer, U. V. Waghmare, N. A. Spaldin, K. M. Rabe, Phys. Rev. B 71 (2005) 014113. 